\title{
Historiographie médiévale et moderne dans le Saint Empire romain germanique
}

Jean-Marie Moeglin

\section{OpenEdition}

\section{Journals}

Édition électronique

URL : https://journals.openedition.org/ashp/1003

DOI : 10.4000/ashp.1003

ISSN : 1969-6310

\section{Éditeur}

Publications de l'École Pratique des Hautes Études

\section{Édition imprimée}

Date de publication : 2 février 2011

Pagination : 171-186

ISSN : 0766-0677

\section{Référence électronique}

Jean-Marie Moeglin, « Historiographie médiévale et moderne dans le Saint Empire romain

germanique ", Annuaire de l'École pratique des hautes études (EPHE), Section des sciences historiques et philologiques [En ligne], 141 | 2011, mis en ligne le 24 février 2011, consulté le 06 juillet 2021. URL: http://journals.openedition.org/ashp/1003 ; DOI : https://doi.org/10.4000/ashp.1003 


\title{
HISTORIOGR A PHIE MÉDIÉVALE ET MODERNE DANS LE SAINT EMPIRE ROMAIN GERMANIQUE
}

\author{
Directeur d'études : M. Jean-Marie MoEgLiN
}

Programme de l'année 2008-2009 : I. L'invention de la guerre de Cent Ans. - II. Les relations entre la France et le royaume d'Allemagne. - III. Questions d'historiographie médiévale et moderne.

\section{L'invention de la guerre de Cent Ans}

L'on a poursuivi au cours de cette année l'enquête visant à proposer une nouvelle lecture de la séquence d'événements dont l'historiographie a fait l'histoire de la guerre de Cent Ans. Le traité de Brétigny-Calais en 1360 peut être considéré comme l'aboutissement d'une stratégie négociatrice obstinément mise en œuvre par Édouard III depuis octobre 1337 : en échange de l'abandon de sa revendication du trône de France, le roi d'Angleterre obtenait la possession en toute souveraineté d'une grande Aquitaine.

Dans les faits, cette paix « finale » de Brétigny-Calais, conçue comme une paix entre deux royaumes et deux rois, était une paix impossible autant pour l'un que pour l'autre des contractants. Édouard III ne s'est jamais résigné à sauter véritablement le pas et à renoncer à son « emprise »; sans doute obsédé par le sentiment de son honneur, il a refusé de faire procéder, en dépit des demandes pressantes du roi de France Jean le Bon, à la double renonciation à laquelle, selon la clause modificative de Brétigny introduite à Calais, les deux rois auraient dû procéder non pas directement à Calais mais un an plus tard à Bruges. Après la mort de Jean le Bon en 1364, son fils Charles V n'aura rien de plus pressé que de revenir sur la concession de l'Aquitaine en toute souveraineté; la « bévue » d'Édouard III lui ouvrait à cet égard un boulevard dans lequel, appuyé sur l'expertise du grand juriste italien Jean de Legnano, il s'est engagé en 1369, provoquant ainsi la reprise de la guerre.

La paix de Brétigny-Calais est néanmoins un événement décisif et un tournant dans l'histoire de « la guerre de Cent Ans racontée par elle-même »; l'idée de l'« emprise » menée par le roi anglais pour la récupération de son droit à la couronne de France et à laquelle son honneur chevaleresque aurait dû lui interdire de renoncer quel qu'en soit le prix, n'est évidemment plus crédible après Brétigny-Calais; certes, formellement, le roi anglais a repris le titre de roi de France qu'il avait cessé de porter pendant quelques années (sans y avoir renoncé) et il prétend à nouveau obtenir la récupération de son droit, mais il est clair que c'est purement formel; dans les faits, la revendication du trône français a totalement disparu des négociations dès qu'elles reprennent à Bruges dans les années 1370 avant de se poursuivre à partir des années 1380 à Leulinghem, sur la frontière entre Calais et Boulogne. Ce qui est désormais sur le devant 
de la scène, c'est l'idée qu'un état de guerre funeste, quelle qu'en soit l'origine, règne entre les royaumes d'Angleterre et de France; ces maux entraînés par la guerre sont insupportables et paralysent la nécessaire défense de la chrétienté contre les infidèles; il faut impérativement y mettre fin; on avait failli y parvenir à Brétigny-Calais; l'on avait été alors tout près du but; il faut reprendre Brétigny-Calais - ce n'est pas un hasard si les négociations reprennent précisément à Bruges, là où l'on devait se réunir en 1361 pour procéder solennellement à l'échange mutuel des renonciations prévu à Calais - et il faut achever Brétigny-Calais comme paix entre les royaumes et leurs rois. Les deux parties y sont prêtes (mis à part quelques moments de fortes tensions voire de reprise de la guerre); il faut simplement trouver la solution technique qui permettra la conclusion d'une paix acceptable par les deux parties, l'enjeu étant non pas la couronne de France mais l'étendue et surtout le statut de l'Aquitaine remise au roi anglais (avec l'idée d'une souveraineté limitée par exemple).

Après avoir paru très proches du succès, ces négociations aboutiront finalement à un échec; l'on s'en tire en 1395 par la conclusion de trêves de longue durée (donc à priori illimitées) qui gèlent la situation en évitant à l'une ou l'autre partie de reculer sur la défense de son droit, et en rétablissant la relation d'amour entre les deux rois, et donc leurs royaumes, par le biais de la rencontre personnelle des deux souverains et surtout du mariage conclu entre Richard II et Isabelle de France, fille de Charles VI, si bien que Charles VI n'est plus pour Richard II son « adversaire de France » mais son père bien aimé contre lequel il ne saurait conduire la guerre... Mais la déposition puis l'assassinat de Richard II en 1399-1400 remettent tout en cause.

\section{Les relations entre le royaume de France et le royaume d'Allemagne au cours des derniers siècles du Moyen Âge}

L'on s'est intéressé cette année à la question de la vision de l'autre.

1. Les obstacles à la connaissance de l'autre n'étaient pas minimes : la distance qui séparait les centres des deux pays et la lenteur des communications sans doute, mais plus encore l'ignorance mutuelle de la langue de l'autre

a. La connaissance de la langue de l'autre est un élément décisif d'une communication réussie. Or, même parmi les souverains allemands, la connaissance du français semble avoir été très limitée. Si Adolf de Nassau, aux dires de Ptolémée de Lucques, savait le français, il n'en était sans doute pas de même avec son prédécesseur Rodolphe de Habsbourg, et en tout cas pas avec son successeur Albert de Habsbourg. Charles IV pouvait parler le français, l'italien, l'allemand, le tchèque et le latin; dans la Bulle d'or, il demande aux princes électeurs de faire instruire leurs fils et futurs successeurs dans les langues étrangères mais il ne mentionne que le latin, l'italien et la langue slave, c'est-àdire les langues que l'on parlait dans l'Empire, à l'exception notable du français.

De fait, différents princes allemands font état de leur ignorance du français. Le 28 mars 1445, le duc de Clèves dans une lettre au roi de France soutient que personne à sa cour ne comprend bien le français. Il est vrai que l'affaire se situe dans le cadre de la Soester Fehde (1444-1449) entre l'archevêque de Cologne, soutenu par le roi de France, et le duc de Clèves, soutenu par le duc de Bourgogne, mais le fait que l'on ait 
pu prendre ce prétexte prouve que cette ignorance n'avait rien d'invraisemblable. Dans la noblesse, la connaissance du français ne pouvait qu'être exceptionnelle; l'on sait que lorsque Charles IV vint à Paris en 1378, il lui fallut traduire aux nobles de sa suite le discours de Charles V car ces derniers «n'entendoient pas françois ». Dans les villes la situation n'était pas meilleure : à Francfort en 1454 une lettre en français arrivée de Metz ne peut être lue car « Und nyemand konde den brieff gelesen ».

Il va de soi que du côté français, la situation était encore bien pire; l'idée qu'un roi de France aurait pu apprendre une langue étrangère et notamment l'allemand serait à coup sûr apparue bien farfelue - Charles VII était pourtant le fils d'une princesse bavaroise - et la diffusion de l'allemand était d'autant plus réduite que, sauf dans le cas particulier de la Flandre, le royaume de France, à l'inverse du royaume d'Allemagne, ne comprenait pas de territoires de langue allemande.

b. Une circulation des personnes entre l'un et l'autre pays somme toute limitée

Vu de loin, l'on pourrait avoir l'impression d'une circulation des personnes à la fois ininterrompue et très variée socialement. L'impression est trompeuse : d'abord cette circulation se faisait beaucoup plus de l'Allemagne vers la France que de la France vers l'Allemagne; ensuite et surtout, elle reste quantitativement très limitée; chaque année séjournaient en France ou passaient par la France quelques dizaines de maîtres et étudiants; quelques dizaines de marchands et d'artisans; quelques pèlerins ou « touristes », au mieux quelques dizaines; quelques diplomates et autres messagers; de manière tout à fait exceptionnelle quelques dames, nobles de cour et autres serviteurs accompagnant une princesse venant épouser un prince français ou allemand; enfin, de manière très irrégulière, quelques centaines voire quelques milliers de gens de guerre dans l'une ou l'autre direction en fonction des spasmes de la guerre de Cent Ans. Les individus concernés avaient ainsi eu la possibilité de se faire sur place une image de l'autre; exceptionnellement, ils ont même pu donner une forme écrite à leur expérience, sous la forme de souvenirs de séjour - par des universitaires tels que Konrad von Megenberg ou Heinrich Toke - ou de récits de voyages et pèlerinages. Il ne s'agit néanmoins pas d'une production massive et, au vu du nombre de manuscrits laissés par ces textes, le nombre de leurs lecteurs ne doit surtout pas être exagéré.

c. Pas de système organisé de diffusion des nouvelles

Un système organisé de diffusion des nouvelles aurait pu dans une certaine mesure permettre de contourner cet obstacle de la langue et de pallier cette relative rareté des contacts humains mais l'on sait qu'il est resté à l'état d'ébauche du Moyen Âge à l'époque moderne, surtout en ce qui concerne la diffusion des nouvelles de pays à pays.

D'un pays à l'autre en revanche, les nouvelles se transmettaient par des canaux plus ou moins hasardeux. La rumeur se fait d'abord messagère des nouvelles importantes mais elle est ensuite relayée, confirmée ou infirmée, par des messagers ad hoc: le roi de France Philippe le Bel déclare avoir appris la nouvelle de la mort d'Albert I ${ }^{\text {er }}$ le $1^{\text {er }}$ mai 1308 d'abord par la rumeur puis par l'assertion de sûrs messagers, ceci dans une lettre écrite à Poitiers le 20 ou le 27 mai 1308.

Derrière la diffusion apparemment « spontanée » - par la rumeur - de ces nouvelles, on peut soupçonner l'existence de contacts de nature diverse, économiques 
ou dus à la présence de voyageurs. L'auteur des annales de Lubeck qui écrit dans les années 1320 se montre étonnamment curieux et informé des nouvelles de France : il rapporte par exemple que le 12 mars 1314 le roi de France a fait brûler le grand maître et le provincial de l'ordre des Templiers, pour prendre leurs richesses, précise-t-il. Il connaît aussi l'histoire de l'adultère des brus du roi de France et il sait que c'est la reine d'Angleterre, fille de Philippe le Bel, qui a conduit le roi là où dormaient les amants adultères. Il rapporte également l'exécution d'Enguerran de Marigny et l'expédition contre les Flamands de 1316. Cet intérêt s'explique en fait très facilement : Lubeck avait des relations très étroites avec la Flandre.

Des sources ultérieures nous montrent plus précisément comment les marchands s'informaient mutuellement des nouvelles politiques importantes. Ainsi, le 5 décembre 1382, Albert Van Halle, clerc du Marchand allemand à Bruges, écrit-il à Lubeck les nouvelles de la campagne du roi de France en Flandre : défaite et mort d'Artevelde à Roosebeke; tentative pour extorquer aux marchands étrangers de Bruges un prêt forcé... Pour les marchands, la politique et les affaires avaient partie liée et il était donc important de diffuser très vite les nouvelles.

Parallèlement à cette diffusion « spontanée » ou « semi-spontanée » des nouvelles, des systèmes de diffusion dirigée des nouvelles commençaient à exister : la propagande princière savait diffuser dans toute l'Europe, sous la forme de « journaux », les nouvelles qu'elle voulait faire connaître : la reprise dans différentes chroniques allemandes de la liste des morts illustres de la bataille de Crécy est ainsi un résultat de l'efficacité de la propagande du roi anglais dont s'est fait le relais le chevalier (autrichien) Johann von Schönfeld; celui-ci commandait une troupe d'hommes d'armes au service d'Édouard III dans la campagne de 1346; blessé dans la bataille, il envoie de Bruges à l'évêque Gottfried de Passau un « journal » sur Crécy pourvu d'une cédule donnant les noms des morts illustres; le tout complété par la prophétie «Lilium » interprétée au profit du roi anglais; c'est ainsi que la liste des morts de Crécy se retrouve dans bien des chroniques allemandes.

La transmission des nouvelles de pays à pays restait néanmoins en tout état de cause fort aléatoire; si les nouvelles importantes, avant tout de nature politique, passaient par des canaux divers et arrivaient ainsi plus ou moins déformées, le tout venant des nouvelles en revanche et des impressions sur le pays voisin et ses habitants restait en revanche dans une large zone d'ombre.

d. Une information lacunaire, réduite aux informations spectaculaires, et souvent tronquée

Cette information ressortit en fait à trois rubriques bien précises : les décès et avènements de nouveaux souverains; relations politiques directes entre les deux pays : guerres, mariages, traités d'alliance; événements situés à proximité immédiate de la frontière.

Seuls les événements spectaculaires sortent de ce cadre de perception : l'épopée impériale de Louis de Bavière en 1328 a laissé dans les sources françaises un écho dont on peut montrer qu'il est bien dû au caractère spectaculaire de l'événement. 
Il en va de même dans l'historiographie allemande. Seuls les événements véritablement sensationnels retiennent l'intérêt; la bataille de Courtrai, retentissante défaite du roi de France, ou encore la destruction de l'ordre des Templiers.

Une information réduite par conséquent aux événements spectaculaires, mais aussi une information déformée et tronquée. Des erreurs sur les noms de lieu et de personne, sans conséquence certes mais tout de même étonnantes, peuvent être commises ; ainsi, la Chronique de Flandre, vers 1340 et, à partir d'elle, les Grandes chroniques de France sont en mesure de retranscrire exactement la lettre de défi envoyé par Adolphe de Nassau à Philippe le Bel mais, dans la datation de la lettre, le transcripteur a mal lu Nuremberg et cette ville est devenue Maubeuge sans doute mieux connue de l'auteur français.

Plus grave, l'explication donnée aux grands épisodes de l'histoire est bien souvent fantaisiste. Ainsi, dans le long récit consacré par le chroniqueur Richer de Senones, en Lorraine de langue française, à la bataille de Bouvines, les causes de la dispute entre Ferrand de Flandre et le roi de France sont évoquées de manière passablement fantaisiste : mis trop souvent échec et mat par sa femme au jeu d'échecs, le comte de Flandre l'aurait violemment frappée et maltraitée, celle-ci du coup aurait été se plaindre à son parent le roi de France et ce dernier aurait menacé le comte de Flandre; c'est ainsi que ce dernier aurait été trouver l'empereur Othon et lui aurait promis de le faire roi à Paris. Le récit des causes de la guerre de Cent Ans est tout aussi fictif chez de nombreux chroniqueurs allemands contemporains : Matthias v. Neuenburg prétend ainsi l'expliquer en évoquant curieusement un enfant de la première femme de Charles IV, né après le divorce, qui pour être préservé aurait été échangé contre un autre mort peu après et qui aurait été confié au roi d'Angleterre pour qu'il l'élève, mais Matthias précise tout de même in fine que le roi d'Angleterre réclame pour lui la couronne de France et non pas pour cet enfant.

e. Un large désintérêt pour le voisin

Cette information lacunaire et souvent tronquée et fantaisiste est-elle vraiement due à l'impossibilité d'obtenir une information précise et concrète sur le voisin? Ne s'agit-il pas plutôt d'un très large désintérêt pour le voisin? Quelques indices nous montrent que les chroniqueurs en savaient peut-être plus, et à coup sûr auraient pu savoir plus, s'ils l'avaient voulu, sur le voisin que ce dont ils font état dans leurs chroniques. Le biographe de Philippe Auguste, Guillaume le Breton, par exemple connaissait la déposition d'Henri le Lion en 1180 mais il ne l'évoque que pour jeter le discrédit sur l'avènement de son fils Othon de Brunswick. Le caractère lacunaire et déformé des informations données sur le voisin pourrait bien s'expliquer par une véritable indifférence pour ce qui se passait chez le voisin, un désintérêt justifiant une réelle désinvolture à l'égard de la connaissance exacte des événements le concernant. Ce n'était pas forcément l'information accessible et obtenue qui déterminait l'image de l'autre mais au moins autant une image préétablie de l'autre qui déterminait l'information que l'on donnait sur lui.

Il n'est pas surprenant dans ces conditions que l'image de l'autre soit largement constituée de clichés et de stéréotypes. 


\section{Une image de l'autre dans laquelle les clichés jouent un grand rôle}

L'étranger, suivant la représentation classique au Moyen Âge, est celui qui se caractérise par des mœurs différentes des siennes. De fait, que les mœurs ne soient pas les mêmes entre les habitants des deux pays paraît un fait établi pour les contemporains; le Mesnagier de Paris donne ainsi quelques conseils à sa jeune épouse en ce qui concerne la réception d'étrangers : il explique que les Allemands reprochent aux Français de ne pas assez faire cuire les carpes, ce qu'ils jugent mauvais pour la santé; s'ils mangent avec des Français des carpes préparées par un cuisinier français, ils font alors recuire leur poisson alors que les Français se régalent de cette carpe mi-crue. Cette différence des coutumes ne concerne pas seulement le domaine de la cuisine mais s'étend aux domaines les plus courants de la vie quotidienne. Le welsche lorrain Richer (†1267), moine de l'abbaye de Senones dans les Vosges, évoque le mos Theutonicorum. Quelques siècles plus tard, le mémorialiste bâlois Hans Knebel rapporte que Charles le Téméraire est venu, à la fin de 1473, loger à Breisach dans l'hôtel de Hans Werner von Pforrs car celui-ci possédait en effet des cheminées qui correspondaient au mos Gallicanum.

a. Une représentation de l'autre réduite à quelques clichés

Mos germanicum contre mos gallicanum : cela poussait attribuer à l'ensemble des habitants du pays voisin quelques traits particuliers qui permettent de lui donner une identité collective; c'est ce que l'on appelle des clichés ou stéréotypes.

- Il existait de fait au Moyen Âge une tradition ancienne de lieux communs et de clichés « nationaux ». Les inventaires de stéréotypes nationaux sont un véritable genre littéraire au Moyen Âge; ainsi conservé dans le manuscrit d'une chronique normande du milieu du $\mathrm{XV}^{\mathrm{e}}$ siècle :

pitié de lombart / labour de picart / loialté d'engloys / larchesse de franchois / humilité de normant / conscience d'allemant / devotion de bourguenon / avec le sens de breton / ces viii choses, par Saint-Bon / ne vallent pas j bouton.

Quelle importance faut-il attribuer à ces clichés ? On y trouve aussi bien la moquerie légère que la remarque blessante et haineuse; mais on peut penser que pendant longtemps il s'agissait plutôt de jeux de plumes et d'exercices scolaires qui servaient simplement à divertir, avec même probablement une certaine dose d'auto-ironie. Toutefois lorsque cette typologie des caractères nationaux se retrouve dans une prophétie fort répandue et mise en rapport avec le massacre des Vêpres siciliennes, Gallorum levitas Germanos iustificabit ..., on peut se demander s'il s'agit encore simplement de jeux de plumes.

— Les clichés « nationaux » comme représentation véridique du caractère et des mœurs de l'autre?

Il se pourrait en effet que les clichés aient pris à partir des $\mathrm{XII}^{\mathrm{e}}-\mathrm{XIII}{ }^{\mathrm{e}}$ siècles plus d'importance. Au début du XIII ${ }^{\mathrm{e}}$ siècle, Jacques de Vitry dans son Historia occidentalis soutient que les rixes qui se produisent sur le pavé parisien naissaient souvent des insultes que les différentes nationalités se lançaient au visage : les Anglais étaient qualifiés d'ivrognes et de gens qui avaient une queue; les Français étaient qualifiés d'orgueilleux, de mous et d'efféminés, les Allemands de furieux et de grossiers ivrognes, les 
Normands de vantards, etc. Réduire le caractère et les mœurs de l'étranger à quelques stéréotypes apparaissait désormais comme un procédé tout à fait légitime.

Les contacts devenus plus fréquents entre peuples, au lieu de permettre de faire apparaître la fausseté ou à tout le moins le caractère sommaire et bien trop général des clichés « nationaux », auraient plutôt contribué à les enraciner et à les légitimer. Le 20 juin 1469 (après le traité de Saint-Omer), l'archiduc Sigismond donne un dîner en l'honneur des envoyés bourguignons qui sont venus à Thann prendre livraison des biens mis en gage en Alsace. Un rapport des envoyés bourguignons se moque de la grossièreté des manières du duc et de ses courtisans. Ils ont tout à fait confirmé ce que l'on savait d'eux par avance.

Alexandre von Roes à la fin du XIII ${ }^{\mathrm{e}}$ siècle entend donner la liste des principales vertus et des principaux défauts des grands peuples de la Chrétienté; il énumère ainsi les principaux stéréotypes négatifs que Français et Allemands étaient susceptibles de se voir reprochés. Les Allemands étaient caractérisés par leur «crudelitas, rapacitas, inurbanitas, discordia ... »; les Français par leur "superbia, luxuria, clamor, garrulitas, inconstantia, se ipsos amare et omnes despicere ». Du moins Alexandre donnaitil un jugement équilibré et équitable sur les défauts des deux peuples. Ses successeurs n'ont pas le même souci de l'équilibre; ils font de leurs défauts prétendus des vertus et ils accablent le voisin. Fondamentalement, les Allemands se considèrent eux-mêmes comme des parangons de vaillance et de courage et ils insistent sur le caractère efféminé et la vanité des Français qui en fait de très mauvais soldats; bien évidemment, les Français se considèrent eux comme l'incarnation du raffinement par rapport aux barbares brutaux que sont leurs voisins.

b. Les clichés et stéréotypes en arrivent à fournir un véritable cadre de perception pour interpréter le déroulement de l'histoire tel que les chroniqueurs pouvaient l'observer. La bataille de Courtrai est un événement spectaculaire qui a eu un grand écho dans l'historiographie allemande. Le récit des chroniqueurs allemands est néanmoins très souvent sommaire. Lorsque il connaît une certaine précision, il se concentre généralement sur un motif central, celui des fossés que les Flamands, trop peu nombreux pour résister à la puissante et orgueilleuse armée française, auraient creusés sur le champ de bataille; la chevalerie française trop sûre de sa force s'y serait précipitée et auraient été facilement égorgée. Le motif est-il véridique? Cela paraît peu probable et fait sans doute plutôt partie des topoi sur le récit des batailles. Pourquoi les chroniques allemandes le reprennent-elles alors à l'unisson? Il permet en fait de montrer que les Flamands, en nombre bien inférieur à celui des Français, l'ont emporté grâce à leur courage et à leur virtus face au nombre et à l'arrogance française. Dans une telle situation en effet, seule la ruse peut permettre de l'emporter suivant les modèles bibliques de David et de Judas Macchabée.

D'autres motifs, à caractère sexuel et bien plus imaginaires encore, sont susceptibles de venir se greffer sur le récit. Jean de Winterthur rapporte le bruit selon lequel des juments en chaleur auraient été placées par les Flamands en haut de la colline; elles auraient ainsi servi d'appâts aux chevaux français qui auraient directement précipité leurs cavaliers dans les fossés remplis d'eau. Belle métaphore de la luxuria qui domine le caractère français et dont Philippe le Bel donnera encore un exemple, en 
forçant, selon l'auteur de la chronique rimée styrienne, les prêtres à épouser les veuves des morts de Courtrai!

Cette représentation de l'autre sous forme de clichés péjoratifs conduit à et soustend une confrontation des deux peuples qui permet de se mettre en valeur soi-même et d'affirmer sa propre identité.

\section{La confrontation entre deux peuples permet de définir et d'affirmer sa propre identité nationale}

Cette confrontation se situe sur l'arrière-plan de l'affirmation des états-nations et de leur identité laquelle n'empêche pas, au moins jusqu'à l'époque des humanistes, la permanence d'une réflexion fondée sur la détermination du bon ordre du monde et de la place que les différents peuples y ont reçue de Dieu sur la base de leur plus ou moins grande dignité et de leurs plus ou moins grands mérites. Elle s'organise ainsi autour de quatre grands thèmes.

a. Un empire universel est-il nécessaire à l'ordre du monde ? Le royaume de France est-il soumis à l'Empire?

Pour les Allemands le fait que leur souverain soit l'empereur universel était une source majeure de fierté et ils ne se privaient pas de de rappeler avec une certaine complaisance que l'empereur, successeur d'Auguste, doit de jure exercer la souveraineté sur l'ensemble du monde. Si Alexandre von Roes reconnaissait du bout des lèvres à la fin du XIII ${ }^{\mathrm{e}}$ siècle qu'il avait été historiquement établi que le roi de France n'aurait juridiquement pas de supérieur, il était assez isolé dans cette position. Au plus fort du conflit entre Louis de Bavière et la papauté, l'ambassadeur de Louis à la Curie, Marquart von Randegg, déplore lors du consistoire du 11 avril 1337 (auquel assistait une délégation française) que l'Allemagne qui est domina gencium et princeps provinciarum propter imperium in ipsum translatum soit tellement troublée par les procès pontificaux.

L'auteur de la chronique rimée styrienne dans le premier quart du XIV siècle rapporte que, sur son lit de mort, saint Louis aurait conseillé à son fils (Philippe le Bel selon le chroniqueur) de faire droit à l'Empire et de recevoir son royaume comme fief de l'empereur. Philippe le Bel très ennuyé aurait réuni ses barons et des experts; ces derniers lui confirment la légitimité de cette demande, ce qui fâche fort les barons (« die Franzois »); ils obtiennent finalement de saint Louis qu'il déclare que cette vassalité ne vaut que pour l'empereur et pas pour le roi des Romains; or il n'y a pas à cette époque d'empereur couronné...

Mais sans doute s'agit-il de prétentions dont le caractère théorique est reconnu; de fait, sur le fond, les théoriciens allemands sont partagés entre le souci de défendre ce qui est la fierté, l'honneur - et aussi le ciment - de la nation allemande, le fait que Dieu lui ait confié la responsabilité de conduire les hommes vers le salut éternel et, d'un autre côté, le souhait de construire un état allemand, un regnum teutonicorum, qui puisse être aussi puissant que ses voisins de l'ouest, le royaume de France et le royaume d'Angleterre. Au milieu du XIV e siècle, Lupold von Bebenburg écrit un traité dont le but est de montrer que le roi allemand élu par les princes à Aixla-Chapelle reçoit ipso facto l'intégralité des droits souverains dans les territoires réel- 
lement soumis à ses prédécesseurs : il est princeps in suis regnis comme n'importe quel autre souverain. Il n'a nul besoin pour cela de l'approbation pontificale. S'il accède à l'Empire, cela ne lui apportera guère plus qu'un nom, tout au mieux quelques droits symboliques.

Le dilemme n'a jamais été véritablement tranché tout au long du Moyen Âge. Hans von Hermannsgrün pouvait encore tenter à la fin du $\mathrm{Xv}^{\mathrm{e}}$ siècle de galvaniser les princes allemands en leur lançant " ad imperandum omnibus gentibus nati estis »; la tirade était certes purement rhétorique, mais la fierté des Allemands était bien d'être les possesseurs de l'Empire, la plus haute dignité temporelle dans le monde.

En France également, indépendamment en fait de toute revendication concrète de quelque empereur que ce soit à une domination universelle et surtout à une autorité quelconque sur le royaume de France, le problème n'a pas cessé de préoccuper les esprits. Tout au long du XIII ${ }^{\mathrm{e}}$ siècle les historiens français se sont demandés comment concilier la reconnaissance de l'Empire romain comme quatrième et dernier empire universel, selon le plan de Dieu, avec l'existence du royaume de France. À la fin du XIII $^{\mathrm{e}}$ siècle et au début du XIV ${ }^{\mathrm{e}}$ siècle, certains historiens et théoriciens français ont trouvé une solution radicale. Pour Johannes Quidort (Jean de Paris) au début du $\mathrm{XIV}^{\mathrm{e}}$ siècle, de même que les empires précédant l'Empire romain se sont effondrés, l'Empire romain va lui aussi s'effondrer et disparaître. Jean de Saint-Victor établit que l'Empire n'est nullement nécessaire à l'ordre du monde; il est au demeurant vacant et destiné à le rester. L'histoire universelle en tout état de cause disparaît du programme de travail des historiens français.

Parallèlement, légistes et plus encore canonistes vont développer des théories qui rendent indiscutable l'exercice du pouvoir souverain par le roi de France à l'intérieur de son propre royaume. Le roi de France est «princeps in suo regno » selon une formule qui apparaît au milieu du XIII ${ }^{\mathrm{e}}$ siècle sous la plume du canoniste Jean de Blanot. L'auteur du Songe du Vergier, un proche du roi Charles V, lequel se souvenait sans doute d'avoir assisté au triomphe de la majesté impériale lors de la diète de Metz en décembre 1356, a soin de réfuter très longuement la prétention de l'Empereur à être « seigneur de tout le monde » même si c'est seulement « de droit » et pas « de fait »; il objecte à cette prétention que l'Empire est une création bien postérieure à l'apparition des monarchies qui représentent l'ordre véritablement voulu par Dieu. Au demeurant le royaume de France peut aussi bien revendiquer le nom d'Empire et son roi le nom d'empereur que son voisin de l'est, car ces deux entités politiques, le royaume de France et l'Empire, résultent tout simplement d'un partage fait sur un pied d'égalité par Charlemagne de son héritage impérial.

Néanmoins les Français restent prudents et méfiants vis-à-vis de l'Empire. Charles IV venant rendre visite à Paris à son neveu le roi de France Charles V en 1378 n'a droit qu'à un cheval noir et non pas blanc, la couleur de la souveraineté. Lorsque Alain Chartier, lors de son ambassade de 1425, s'adresse à Sigismond, il ne manque pas d'exprimer l'admiration et la reconnaissance du roi de France pour la majesté de l'Empire voulu et fondé par Dieu, mais il se garde bien de le présenter comme une domination universelle, et il demande à l'empereur en retour de manifester à l'égard du royaume de France une égale estime justifiée, selon Chartier, par l'élection 
divine des rois de France. Le Regnum et l'Imperium sont deux configurations politiques situées sur un pied d'égalité.

b. À qui appartient l'Empire?

Le débat pouvait se déplacer sur un autre terrain; la décrétale Venerabilem du pape Innocent III avait évoqué la translation par la papauté de l'Empire des Grecs aux Allemands. Mais cette translation était-elle forcément la dernière ? Si le pape était l'auteur de cette translation, ne pouvait-il pas exercer à nouveau son droit au profit d'un autre peuple? Les Français n'étaient-ils pas eux aussi habilités à le revendiquer et à demander de quel droit l'Empire devrait appartenir aux Allemands ?

En France, à la fin du XIII ${ }^{\mathrm{e}}$ siècle, parallèlement à la mise en cause de la nécessité de l'Empire, l'idée se fait jour que les Allemands ne sont plus dignes de cette dignité. Ainsi Guillaume de Nangis souligne-t-il que ce n'est pas seulement dans le domaine des armes que le royaume de France l'emporte sur les autres royaumes; il rappelle que le royaume de France a été honoré par Dieu de la translation des trois grâces que sont la foi, la sagesse et la chevalerie. En cette même fin du XIII ${ }^{\mathrm{e}}$ siècle le juriste et publiciste Pierre Dubois exalte également avec conviction le royaume de France comme l'État parfait auquel le monde entier gagnerait à être soumis s'il veut accéder au salut éternel et il développe un plan ambitieux de conquête du monde par le roi de France, une conquête largement pacifique au demeurant; le royaume d'Allemagne notamment sera obtenu par traité. Ce n'est pas un hasard si c'est à la même époque que se situent les plans les plus concrets d'accession du roi de France ou d'un de ses parents à l'Empire.

Dès cette époque cependant, et beaucoup plus nettement à partir des années 1330, le roi anglais va se profiler comme une menace mortelle pour le roi et le royaume de France. L'heure n'était assurément plus parfaitement favorable pour une revendication de l'Empire par le roi de France; l'auteur du Songe du Vergier n'entendait cependant pas exclure qu'il puisse en être ainsi un jour car la propriété de l'Empire par les Allemands n'était nullement un droit éternel et intangible « et aussi pourret au jour [d'huy] le roy de France estre Impereur, ou aultre de la couronne de France, ou d'aultre lignie ». Il faudra néanmoins attendre la fin du $\mathrm{Xv}^{\mathrm{e}}$ siècle pour qu'un roi de France débarrassé de ses grands rivaux, le roi d'Angleterre et le duc de Bourgogne, puisse à nouveau sérieusement envisager de recevoir la couronne impériale.

Néanmoins, dès la fin du XIII ${ }^{\mathrm{e}}$ siècle et le début du XIV ${ }^{\mathrm{e}}$ siècle, les Allemands sont sur la défensive; ils ont conscience que cette possession de l'Empire qui fait leur fierté n'est pas forcément un acquis définitif. Il leur faut faire face aux prétentions d'autres nations, notamment de la nation française, à assumer véritablement la tâche de guider les hommes vers le salut éternel en recueillant l'héritage de l'Empire. Alexandre von Roes écrivait ses traités pénétré de cette crainte et, deux siècles plus tard, le songe de Hans von Hermannsgrün (1495) commence par le récit d'une discussion que l'auteur a eu avec son hôte au sujet des destinées de l'Empire; il avoue que lui et son hôte étaient plus pénétrés de la crainte "de imperio nostro amittendo quam spei de retinendo »; plus loin, il étaye cette crainte : le cours des choses a souvent enseigné que l'Empire était toujours transféré « ab ignavis et non idoneis ad strennuos et maxime idoneos ». 
c. L'histoire de deux peuples : à qui appartient l'héritage des Troyens et des Francs?

Derrière le débat sur la légitimité de la propriété de l'Empire par les Allemands se dissimulait en fait un autre débat : quel est le peuple élu auquel Dieu a voulu confier cette tâche essentielle au salut de l'humanité? S'agissait-il des Français ou des Allemands en tant qu'héritiers des Francs? Plus concrètement, le refondateur de l'Empire, Charlemagne, celui au profit duquel Dieu avait recréé l'Empire romain était-il allemand ou français?

— Quels sont les héritiers et les descendants des Francs?

Le roi de France s'intitulait « rex Francorum » que l'on traduisait par « roi des Français »; pour les Français, il allait donc de soi que les Francs étaient leurs ancêtres directs parmi lesquels se trouvaient Clovis et de Charlemagne.

Pour les Allemands le problème était précisément là : l'héritage franc était aussi celui de Charlemagne et l'on ne pouvait ainsi l'abandonner aux Français. Un récit d' " origo » élaboré et diffusé à partir du $\mathrm{XII}^{\mathrm{e}}$ siècle va leur permettre de disputer aux Français la qualité de descendants directs des Francs et d'établir le peuple allemand comme héritier direct des Troyens venus s'installer à l'est du Rhin, ayant reçu le nom de Francs puis plus tard celui de Theutonici ou de Germani, tandis que les Français n'étaient qu'une souche cadette, bâtarde et l'on ne devrait pas les appeler « Francs » mais «Francigenae » ou mieux «Galli ». Diffusée par les chroniques de Frutolf de Michelsberg et de Godefroi de Viterbe au XII ${ }^{\mathrm{e}}$ siècle, cette histoire va se retrouver chez de nombreux auteurs du XIII ${ }^{\mathrm{e}}$ au XV $\mathrm{XV}^{\mathrm{e}}$ siècle. Les humanistes alsaciens en sont les derniers héritiers avant que la découverte de la Germanie de Tacite ne change vraiment la donne.

— Le personnage de Charlemagne

Le fait de chercher les descendants directs des Francs chez les Français ou les Allemands avait une conséquence importante : selon la version adoptée, Charlemagne était un Français ou un Allemand car on ne pouvait pas lui disputer sa qualité de Franc. C'était à coup sûr un enjeu important, peut-être même le principal enjeu de la dispute autour de l'héritage des Francs.

Le royaume de France comme l'Empire allemand se considéraient en effet comme les héritiers de Charlemagne et les dépositaires de son potentiel de légitimité. Face aux prétentions françaises à l'héritage de Charlemagne roi de France avant d'être empereur, les Allemands ont paru parfois incertains de leur cause; Wolfram von Eschenbach faisait de Charles un Franzoys. Mais déjà la canonisation de Charles en 1165 devrait avoir été une réponse à la récupération de Charles par les rois français. Charlemagne fit ensuite l'objet d'un culte dans de très nombreuses églises allemandes en même temps qu'il était revendiqué comme fondateur et comme ancêtre par de nombreuses églises et dynasties. Peu d'années après la canonisation de Charles par Barberousse, le chroniqueur attitré des Staufen, Godefroi de Viterbe, fidèle à sa version de l'histoire des Francs et des Allemands, réaffirma la germanité de Charles : son père était allemand; lui-même était né à Ingelheim. Lorsque le Franc Charlemagne avait reçu l'Empire, c'était donc bien sûr un Allemand qui avait hérité de l'Empire. Lupold de Bebenburg avait frappé la formule canonique : la translation avait été faite « in personam Karoli 
magni ad reges Francorum et ad Germanos », parce que Charlemagne était un Germain ou plus exactement un «Francus germanicus».

Dans le songe de Hans von Hermannsgrün à la fin du $X^{\mathrm{e}}$ siècle les trois personnages qui apparaissent devant les Grands de l'Empire médusés sont Othon I ${ }^{\mathrm{er}}$ " Germanorum decus et gloria », Frédéric Barberousse « qui labentem rempublicam Germanorum restituit », mais d'abord Charlemagne qui «a arraché l'empire Romain aux Grecs, l'a affermi chez les Allemands et l'a augmenté ». Tous les trois sont guidés par l'amor Romani imperii, quod sanguine nostro Germanis comparavimus. Et en 1501, dans sa Germania, Wimpheling entend démontrer que depuis Charlemagne inclus, et même, avant lui, depuis Pépin le Bref, tous les empereurs ont été des Allemands.

Cette volonté de défendre coûte que coûte la germanité de Charlemagne et de ne pas se limiter à l'exaltation d'Othon $\mathrm{I}^{\text {er }}$ correspond en fait à la volonté d'écrire l'histoire de l'Empire comme l'histoire d'un imperium teutonicorum; un projet déjà bien articulé chez Heinrich von Herford vers 1360; elle vise à identifier de manière indissociable l'histoire de l'Empire refondée par Charlemagne et l'histoire des Allemands et à en faire le fondement d'un imaginaire national. En ce sens, elle n'est pas foncièrement dirigée contre les prétentions françaises mais répond d'abord à la nécessité pour les Allemands de se constituer un imaginaire national qui les unisse.

d. Qui a le meilleur système politique?

Le Regnum appartient aux Français mais l'Imperium reste aux Allemands. La différence de mode d'accès à la dignité suprême apparaît alors comme le symbole même de la différence entre l'Imperium et le Regnum. Au milieu du $\mathrm{XV}^{\mathrm{e}}$ siècle, la traduction de la chanson de geste Loher und Maller, peut-être par la comtesse Elisabeth von Saarbrücken-Nassau, raconte comment la France et l'Allemagne se sont séparées en adoptant deux systèmes différents d'accession au pouvoir souverain : Loher est le fils cadet de Charlemagne; après la mort de son père, son frère Louis (le pieux) refuse de le reconnaître comme héritier mais le conflit entre les deux frères est tranché par le pape : Louis restera roi de France et Loher devient empereur de Rome. Finalement, après quelques autres aventures, le pape règle définitivement l'ordre du monde : la France reste une monarchie héréditaire, l'empire devient une monarchie élective dans laquelle on élira souverain celui qui est le meilleur et le plus fort.

Les Français considéraient bien sûr que l'hérédité était beaucoup plus noble que l'élection; c'est ce que Robert d'Artois, frère de saint Louis, aurait écrit pour refuser une offre pontificale de recevoir l'Empire. La thèse de la supériorité de l'hérédité sur l'élection pouvait en tout cas s'appuyer sur la démonstration fournie par Gilles de Rome dans son très influent De regimine principum. Les Allemands étaient d'un avis tout à fait différent. Alexandre von Roes considère que l'hérédité pratiquée en France est un système moins noble que l'élection pratiquée dans l'Empire. Conrad de Megenberg réfute les arguments de Gilles de Rome en faveur de l'hérédité et il vante la supériorité de l'élection en admettant néanmoins que cela n'est vrai que s'il ne s'agit pas d'une élection corrupta; comme c'est le cas actuellement en Allemagne, il faut reconnaître que le royaume de France et le royaume d'Angleterre, dont les princes sont héréditaires, ont provisoirement un bien meilleur État que le regnum romanorum dont les princes sont élus. De fait, au $\mathrm{XV}^{\mathrm{e}}$ siècle les Allemands devaient convenir 
qu'une réforme de l'Empire était nécessaire faute de quoi celui-ci risquait d'échapper à la nation allemande, mais ils n'en restaient pas moins des partisans résolus de la supériorité de l'élection sur l'hérédité.

Les points de confrontation existaient par conséquent très clairement entre ce que l'on peut appeler l'idéologie nationale française et l'idéologie nationale allemande. L'intensité de cette confrontation ne doit cependant pas être surestimée. À dire vrai, il n'y a pas véritablement de controverse directe entre Français et Allemands; les Français se contentent d'affirmer que l'Empire est un royaume comme les autres, moins prestigieux à dire vrai que le royaume de France. Leur préoccupation est de construire une histoire glorieuse de la France et des Français telle qu'elle se confond avec l'histoire de ses rois depuis saint Clovis. Quant aux Allemands, leur insistance à exposer que l'Empire appartient aux Allemands est-elle vraiment une réponse à la volonté d'autres nations de leur prendre cet Empire ? Ce qui s'exprime fondamentalement, c'est la volonté de construire une histoire allemande.

La confrontation hostile entre Français et Allemands pourrait bien ainsi être plus apparente que réelle. Fondamentalement elle répond à des préoccupations internes bien plus qu'à une volonté agressive à l'égard du voisin. Ne pouvait-elle pas cependant se greffer sur l'émergence d'un véritable sentiment d'hostilité entre Français et Allemands à la faveur des péripéties politiques de trois siècles? La reprise sur un mode incontestablement plus agressif et l'infléchissement des thèmes de cette confrontation chez les humanistes des deux pays à la fin $\mathrm{du} \mathrm{Xv}^{\mathrm{e}}$ siècle n'en étaient-ils pas la preuve?

\section{Français et Allemands sont-ils en passe de devenir des ennemis héréditaires au cours des derniers siècles du Moyen Âge?}

Derrière l'affirmation de soi et, ce faisant, la confrontation limitée, on l'a vu, de deux histoires, peut-on voir se profiler la construction de la figure de l'étranger comme ennemi héréditaire?

L'abbé Gervais de Prémontré écrit au pape Innocent III en 1216 qu'il vaut mieux à la croisade séparer Français et Allemands car ils n'ont jamais pu s'entendre. Rien ne prouve cependant que cette hostilité entre Allemands et Français ait été plus profonde que les traditionnelles échauffourées qui se produisaient entre gens de provinces différentes à l'intérieur d'un même pays, en France comme en Allemagne.

$\mathrm{La}$ « solidification » des États nationaux à partir des XIV $-\mathrm{XV}^{\mathrm{e}}$ siècle pouvait néanmoins déboucher sur l'idée d'un combat des nations, lorsque, par exemple, le roi Édouard III accusait les Français de vouloir détruire la nation anglaise. La confrontation entre Français et Allemands aurait-elle pu alors déboucher sur une haine mortelle entre les Français et les Allemands, d'une lutte à mort entre la nation française et la nation allemande?

La situation n'était pas la même en tout état de cause dans l'un et l'autre pays. Pour les Français, les Allemands ne pouvaient être leurs ennemis héréditaires et ceci pour une raison simple : la place était déjà prise par les Anglais. Les quelques épisodes de conflit ouvert entre les rois de France et d'Allemagne - les déclarations de guerre d'Adolphe de Nassau, de Louis de Bavière et de Sigismond de Luxembourg - n'ont guère été suivis d'effet et n'ont laissé que des traces limitées. L'épisode le plus notable 
est à cet égard le récit légendaire du différend entre Philippe le Bel et Adolphe de Nassau tel qu'on le trouve dans les Grandes Chroniques de France. Ce récit rapporte la fière mais imaginaire réponse que le roi de France aurait faite à la lettre de défi que le roi Adolf de Nassau lui avait fait porter : « Il [Adolf] brisa le seel de la lettre qui moult estoit grande; et quant elle fu ouverte, il n'i trouva riens escript, fors : "Troup alemant." ». L'interjection « troup » est en fait une déformation de l'interjection " Ptrut » qui est bien attesté en ancien français pour exprimer, assez grossièrement, à un interlocuteur un très profond mépris. Sous le coup de l'alliance conclue par Sigismond en 1415 avec le roi d'Angleterre Henri V, l'humaniste parisien Jean de Montreuil rappelle cet épisode et surtout recense soigneusement toutes les raclées qu'un roi de France a pu flanquer à un empereur allemand au cours de l'histoire, mais il est clair que c'est aux Anglais que sa haine va prioritairement.

Pour les Allemands en revanche, les Français n'étaient-ils pas cet ennemi héréditaire? Dietrich von Nieheim recopie quelques vers de Godefroi de Viterbe relatifs à l'affrontement entre le pape Calixte II et l'empereur Henri V et il commente de son propre chef : «nota antiquum odium regalium Francie contra imperium ». De fait, on peut retrouver chez les chroniqueurs allemands le dossier d'un contentieux entre Français et Allemands qui grossit du XIII ${ }^{\mathrm{e}}$ au XV $\mathrm{XV}^{\mathrm{e}}$ siècle. Les grands thèmes en sont :

- la lutte des Anjou et des Staufen représentée comme une lutte des Français contre les Allemands;

— l'échec de la réconciliation de Louis de Bavière et du pape dont le roi de France et les Français seraient responsables;

- la volonté tenace du roi de France de s'assurer le contrôle de la papauté, déjà bien marquée lors de l'affrontement entre Louis de Bavière et la papauté et également fort claire au moment du Grand Schisme;

- la volonté française de prendre l'Empire aux Allemands; c'est une rumeur qui court et qui revient à de nombreuses reprises, alors même qu'aucun plan concret de ce type n'est ni attesté ni même envisageable;

- enfin, la volonté française de s'approprier non seulement la dignité impériale mais aussi les terres de l'Empire.

Les différentes pièces de ce dossier, tout particulièrement les deux dernières, alimentent ce que l'on peut appeler un « Bedrohungsszenario » qui devient au cours du $\mathrm{XV}^{\mathrm{e}}$ siècle un thème de propagande mobilisable à la demande et adaptable à différentes situations.

En 1444 ce sont les villes d'Empire tout particulièrement qui se voient en cibles désignées des attaques du roi de France. La rumeur des prétentions que Charles VII aurait élevées sur les territoires allemands se répand rapidement. Il est douteux qu'il ait fallu voir dans ces déclarations beaucoup plus que la reprise par un souverain en mal de légitimation pour une expédition de circonstance, de la rhétorique sur la souveraineté royale et l'inaliénabilité des terres du royaume à laquelle la guerre de Cent Ans avait habitué les rois de France; mais l'on peut comprendre que les récipiendaires de ces lettres n'aient guère été rassurés!

Par la suite, Wimpheling reprendra cette tradition selon laquelle les Français auraient voulu en 1444 annexer toute la rive gauche du Rhin. La France était ainsi devenue l'ennemi héréditaire des Allemands : en 1498, Maximilien l'avait clairement 
affirmé aux états de l'Autriche antérieure : il réclamait leur soutien contre la France « uwer natturlicher vynnd ».

Faut-il pour autant véritablement conclure au développement d'une haine profonde entre les Français et les Allemands? Ces querelles de princes trouvent certes un prolongement dans des guerres de plume entre humanistes tels que celle que se livrent Robert Gaguin et Wimpheling à propos du « Brautraub » dont Anne de Bretagne aurait été la victime. Toutefois, en l'absence de contacts réguliers et étroits entre Français et Allemands, l'on constate que les épisodes d'hostilité véritablement marquée des Allemands à l'égard des Français paraissent malgré tout rares et dus à des contextes bien spécifiques. Les cas d'hostilité les plus marquées sont à l'évidence dus aux déprédations bien réelles que des troupes venues de l'autre pays font à demeure, même si, comme dans l'affaire des Armagnacs de 1444, elles ont en fait été appelées par les princes allemands. La réaction des villes est significative lorsque des princes allemands demandent qu'un laisser-passer soit accordé à des envoyés du roi de France en octobre 1444, elles refusent catégoriquement : nach solichem sweren handel, den das fromde volk in disem lande begangen, so gebüre uns das nit zu tun. L'émotion et la colère des villes contre le roi de France était compréhensibles mais on aurait tort d'en conclure à un sentiment de haine généralisée à l'égard des Français. Une invectiva contra Armacenos composée sans doute à l'hiver 1444 par un Alsacien ayant fréquenté l'université et les milieux intellectuels d'Heidelberg s'en prend certes violemment au Dauphin et aux Armagnacs, mais elle n'épargne pas non plus l'Empereur, les princes et prélats allemands, et elle ne contient pas d'attaques xénophobes contre les Français en tant que tels. Au demeurant les écorcheurs haïs, non sans raison, par les populations ne sont pas appelés « Français » mais toujours désignés comme étant des gens de guerre plus ou moins en rupture de ban. C'est la soldatestque en rupture de ban que l'on redoute et déteste bien plus que les Français.

Les guerres du Téméraire ont peut-être provoqué une certaine poussée anti-welsche mais en tout état de cause il ne faudrait pas exagérer son intensité : elle vise le Téméraire, « Grand Turc d'Occident » comme l'a montré Claudius Sieber-Lehmann, avant de viser les welsches et les Français. À Berne à l'époque des guerres bourguignonnes c'était le parti pro-français regroupé autour de Niklaus von Diesbach qui domine clairement la politique de la ville même si l'argent du roi de France n'y est pas totalement étranger.

Dans le grand conflit dynastique des maisons d'Autriche et de France qui se dessine à la fin $\mathrm{du} \mathrm{XV}^{\mathrm{e}}$ siècle, les antagonismes nationaux ne sont encore qu'un prétexte complaisamment avancé par les princes lorsque leurs intérêts dynastiques le rend utile et aussitôt abandonnés dès qu'il n'en est plus ainsi ; il est incontestable qu'ils rencontrent néanmoins un écho chez les élites françaises et allemandes et notamment chez les humanistes travaillant à forger les concepts qui, beaucoup plus tard, lorsque l'ordre dynastique se délitera, canaliseront l'essor du nationalisme contemporain. On en était encore très loin à la fin du Moyen Âge.

\section{Questions d'historiographie médiévale et moderne}

$\mathrm{M}^{\mathrm{me}}$ Nicole Pons, ingénieur de recherches au CNRS, nous a fait l'honneur de présenter sa nouvelle édition d'une « épopée latine humaniste concernant Jeanne d'Arc 
et le siège d'Orléans » conservé dans l'un des manuscrits du procès en nullité de la condamnation de Jeanne d'Arc, et $\mathrm{M}^{\mathrm{me}}$ Isabelle Guyot-Bachy, maître de conférences à l'université Paris-12 - Val-de-Marne nous a également fait l'honneur de présenter un exposé sur le thème «La guerre de Cent ans : la faute aux Flamands?». 